\title{
Value of electron microscopy in diagnosis of renal disease
}

\author{
J M Pearson, L J McWilliam, J D Coyne, A Curry
}

\begin{abstract}
Aims-To assess the role and value of electron microscopy in the diagnosis of renal disease.

Methods-Retrospective evaluation of 88 renal biopsy specimens received for primary diagnosis by assessment of the contribution of electron microscopy to the final diagnosis in the knowledge of the light microscopy and immunofluorescence findings.

Results-Electron microscopy had an important diagnostic role in $75 \%$ of cases and was essential or necessary for diagnosis in $25 \%$. In $25 \%$ of cases electron microscopy was considered unhelpful in diagnosis.

Conclusion-Electron microscopy has an integral role in the diagnosis of renal disease, and tissue should be taken for electron microscopy in all cases if possible. In some selected cases once the light microscopy and immunofluorescence findings are known it may be possible to forego electron microscopic examination. Electron microscopy is particularly useful in the differential diagnosis of minimal change disease and the nephrotic syndrome.
\end{abstract}

(F Clin Pathol 1994;47:126-128)

Electron microscopy has been used in the pathological diagnosis of renal glomerular disease over the past three decades and its diagnostic value has been strongly emphasised. However, few recent reports have critically assessed the role of electron microscopy. Its usefulness in other diagnostic areas, such as tumour pathology, has declined considerably and this along with inevitable financial pressures to reduce expensive investigations prompted us to perform this study.

We receive about 100 renal biopsy specimens every year, which are mostly performed for primary diagnosis. Immunofluorescence and electron microscopic examination are routinely performed in all cases. This study was undertaken to assess the value of this approach in the investigation of renal pathology with particular reference to electron microscopy, and to assess whether a more selective approach could be adopted.

Methods

All the renal biopsy specimens taken in 1990 were retrieved from file and were reviewed by the reporting pathologists ( $\mathrm{LMcW}$ and JDC). The age range of the patients in this study was 15-74 years with a mean age of 49 years. Cases in which electron microscopy was unsatisfactory due to absence of glomeruli were excluded.

A total of 88 cases were re-examined and an assessment made of the contribution of electron microscopy to the final diagnosis. In 79 cases biopsy was undertaken for the investigation of primary renal disease and the remaining nine cases were transplant biopsies. The assessments were made by the reporting pathologists who re-examined the light microscopy slides and immunofluorescence pictures and formulated their provisional diagnosis without taking into account the clinical data. The immunofluorescence technique used in all cases was a standard direct method using $6 \mu \mathrm{m}$ frozen sections fixed in acetone and stored at $-20^{\circ} \mathrm{C}$ until stained.

Commercial antisera to IgG, IgA, IgM, $\mathrm{C} 3, \mathrm{Clq}$ and fibrinogen were used and known positive controls included in each run, plus negative controls, the antisera being replaced by buffer. Photomicrographs of positive findings were kept in all cases. Immunoperoxidase studies were not performed. The electron microscopy pictures were then reviewed and an assessment made as to whether electron microscopy was essential, helpful, or unhelpful in establishing the final diagnosis.

The contribution made by electron microscopy was divided into the following categories:

(A) Essential-The diagnosis could not have been made without electron microscopy. Electron microscopy findings confirmed the light microscopy and fluorescence findings and was necessary to establish a precise diagnosis and exclude other possibilities.

(B) Helpful-Electron microscopy findings were entirely consistent with the diagnosis made by light and fluorescence findings but were not fundamental to making the final diagnosis.

(C) Unhelpful-Electron microscopy findings were not of any help in establishing the diagnosis.

The clinical presentation was subsequently separately assessed in relation to the electron microscopy contribution to the final diagnosis and an assessment was also made as to whether the electron microscopy findings in each case were predictable from the light 
microscopy and immunofluorescence findings, taking into account the clinical data.

\section{Results}

The diagnostic categories and the contribution of electron microscopy are shown in table 1. The predictability of the electron microscopy findings, based on the light microscopy and fluorescence findings related to diagnosis, is shown in table 2 . The clinical

Table 1 Contribution of electron microscopy (EM) to diagnostic categories

\begin{tabular}{|c|c|c|c|c|}
\hline \multirow[b]{2}{*}{ Categories/diagnosis/final diagnosis } & \multicolumn{4}{|c|}{ E M Category } \\
\hline & $A$ & $B$ & $C$ & Total No of cases \\
\hline $\begin{array}{l}\text { Minimal change } \\
\text { Membranous GN } \\
\text { Mesangial proliferative GN IgA } \\
\text { Mesangial proliferative GN IgM } \\
\text { Mesangiocapillary GN } \\
\text { Crescentic GN } \\
\text { Proliferative GN (SLE) } \\
\text { FSGS } \\
\text { Diabetic nephropathy } \\
\text { Ischaemic/benign hypertension } \\
\text { Malignant hypertension } \\
\text { End stage disease } \\
\text { Amyloidosis } \\
\text { ATN } \\
\text { Tubulointerstitial nephritis } \\
\text { Transplant biopsy } \\
\text { Large vessel thrombosis } \\
\text { Normal } \\
\text { Total }\end{array}$ & $\begin{array}{l}6 \\
1 \\
1 \\
1 \\
2 \\
3 \\
1\end{array}$ & $\begin{array}{l}4 \\
9 \\
4 \\
2 \\
8 \\
1 \\
4 \\
4 \\
3\end{array}$ & $\begin{array}{r}6 \\
1 \\
5 \\
1 \\
1 \\
2 \\
1 \\
1 \\
19\end{array}$ & $\begin{array}{r}6 \\
5 \\
9 \\
5 \\
2 \\
10 \\
3 \\
4 \\
7 \\
10 \\
1 \\
5 \\
3 \\
1 \\
1 \\
9 \\
1 \\
6 \\
88\end{array}$ \\
\hline
\end{tabular}

GN = Glomerulonephritis; SLE = systemic lupus
necrosis; FSGS = focal segmental glomerulosclerosis

Table 2 Predictability of electron microscopy in final diagnosis

\begin{tabular}{|c|c|c|c|}
\hline \multirow[b]{2}{*}{ Final diagnosis } & \multicolumn{3}{|c|}{ Electron microscopy predictability } \\
\hline & Yes & No & Total \\
\hline Minimal change & 6 & & 6 \\
\hline Membranous glomerulonephritis & 5 & & 5 \\
\hline Mesangial proliferative glomerulonephritis IgA & 9 & & 9 \\
\hline Mesangial proliferative glomerulonephritis IgM & 3 & 2 & 5 \\
\hline Mesangiocapillary glomerulonephritis & 2 & & 2 \\
\hline Crescentic glomerulonephritis & 5 & 5 & 10 \\
\hline Proliferative glomerulonephritis (SLE) & 1 & 2 & 3 \\
\hline Focal segmental glomerulosclerosis & 4 & & 4 \\
\hline Diabetic nephropathy & 7 & & 7 \\
\hline Ischaemic/benign hypertension & 9 & 1 & 10 \\
\hline Malignant hypertension & 1 & & 1 \\
\hline End stage disease & 5 & & 5 \\
\hline Amyloidosis & 3 & & 3 \\
\hline Acute tubular necrosis & 1 & & 1 \\
\hline Tubulointerstitial nephritis & 1 & & 1 \\
\hline Transplant biopsy & 3 & 6 & 9 \\
\hline Large vessel thrombosis & 1 & & 1 \\
\hline Normal & 3 & 3 & 6 \\
\hline Total & 69 & 19 & 88 \\
\hline
\end{tabular}

SLE = systemic lupus erythematosus.

Table 3 Clinical presentation in relation to electron miscroscopy category

\begin{tabular}{lcccc}
\hline & \multicolumn{5}{c}{ Electron microscopy category } \\
\cline { 2 - 5 } Clinical presentation & $A$ & $B$ & $C$ & Total (No of cases) \\
\hline Nephrotic syndrome & 8 & 6 & 1 & 15 \\
Proteinuria (non-nephrotic) & 1 & 6 & 7 & 16 \\
Proteinuria and haematuria & 5 & 7 & 5 \\
Haematuria & 1 & 4 & 5 & 11 \\
Acute renal failure & 2 & 6 & 5 & 15 \\
Chronic renal failure & 3 & 1 & & 4 \\
Microscopic haematuria & 4 & 2 & & 6 \\
Transplant & 2 & 1 & & 3 \\
$\downarrow$ Function & 4 & 2 & & 6 \\
Proteinuria & 30 & 43 & 15 & 88 \\
Not specified & & & & \\
Total & &
\end{tabular}

Function $=$ renal function . presentation in relation to the electron microscopy categories is shown in table 3.

Of the 88 cases, electron microscopy had an essential role in 22 and a helpful role in 47. In 19 cases electron microscopy was considered unhelpful. It was possible to predict retrospectively the electron microscopy findings in 69 cases.

\section{Discussion}

The role of electron microscopy in renal biopsy diagnosis is well established and has been the subject of several publications. ${ }^{1-8} \mathrm{We}$ found no publication in the past decade, however, reviewing the role of electron microscopy in renal biopsy diagnosis.

In 1969 Muehrcke et al, ${ }^{1}$ having evaluated a series of 179 cases, found that in only $6 \%$ of cases did electron microscopy contribute significantly to the diagnosis. They concluded that electron microscopy need not be performed as a routine procedure and that an electron microscopy study of renal tissue is a clinical luxury rather than a necessity.

Tighe and Jones ${ }^{2}$ described the electron microscopy findings in a series of 100 cases and concluded that routine diagnostic electron microscopy is of greatest value in the differential diagnosis of the nephrotic syndrome. They emphasised that the main limitations of routine electron microscopy are that it is costly and time consuming. Ben-Bassat $e t a l^{3}$ evaluated routine electron microscopy in the differential diagnosis of the nephrotic syndrome in 37 cases and found that it was necessary for accurate diagnosis, particularly of minimal change disease as opposed to early membranous disease, especially in terms of treatment and prognosis. Dische and Parsons ${ }^{4}$ described the contribution of immunofluorescence and electron microscopy to the diagnosis of glomerulonephritis in 134 cases and concluded that for accurate diagnosis it was essential to supplement light microscopy by one, or preferably both, of these methods. Spargo 5 evaluated the practical use of electron microscopy in the diagnosis of glomerular disease and concluded that there could no longer be any question as to the practical value of electron microscopy and that it should be used wherever a renal biopsy was to be critically evaluated. Siegel et al ${ }^{6}$ evaluated the use of routine electron microscopy in 213 renal biopsy specimens and concluded that electron microscopy contributed to diagnosis or patient management in $48 \%$ of cases. They found that in roughly one in 10 cases biopsied, electron microscopy resulted in a substantially different diagnosis than that suggested by light microscopy alone, but that electron microscopy was useful, in that definitive confirmation of the light microscopic diagnosis was important in precise diagnosis and in management. They pointed out that as it is usually not possible to predict on light microscopy alone those instances where electron microscopy would be of most benefit, this method of examination should be used on a routine basis. Collan 
et $a l^{7}$ re-embedded into resin a series of renal cases from 1967 when light microscopical diagnosis alone was available and performed electron microscopy retrospectively. They showed that electron microscopy made a significant contribution to the precise diagnosis of glomerular disease, because it allowed immune deposits to be detected.

Skjorten and Halvorsen ${ }^{8}$ evaluated the use of semi-thin resin sections and electron microscopy in renal biopsy diagnosis and found that electron microscopy altered the diagnosis in $34 \%$ of cases and yielded additional useful information in another $45 \%$ of cases of glomerulonephritis. They concluded that electron microscopy should be used routinely in suspected cases of glomerulonephritis. Its value in other renal disease was less clear and its use should be decided according to available resources.

In the present study the routine use of electron microscopy, in conjunction with the light microscopic and immunofluorescence findings, was found to have an essential role in $25 \%$ of cases and a helpful role in a further $50 \%$ of cases. In about $25 \%$ of cases electron microscopy was considered non-contributory in making the diagnosis. These figures are comparable with those cited, although some of the previous studies did not include any immunofluorescence findings when making their assessment.

Electron microscopy was considered most useful in the current study in both the diagnosis of minimal change nephropathy and in its differential diagnosis (table 1). In many cases where electron microscopy was considered essential this was by excluding other possibilities-for example, very early membranous disease, early amyloid, or an abnormal basement membrane in normal looking glomeruli by light microscopy. It was also essential in excluding the presence of immune deposits. In such cases the final diagnosis was made by electron microscopy and these were clinically important diagnoses and not simply of academic interest. Electron microscopy was also an important adjunct in cases of clinical nephrosis and in those cases presenting with proteinuria (table 3 ). In many cases where electron microscopy was considered helpful this was in detecting the location and nature of deposits or abnormal matrix material and was complementary to confirming the final diagnosis. However, the electron microscopy findings were considered highly predictable in this area (table 2). The assessment of predictability was made after the electron microscopy findings were knownretrospectively-and also taking the clinical data into consideration. Although it was possible to predict the electron microscopy findings in about $75 \%$ of cases, a prospective analysis of the predictability of the electron microscopy findings may considerably reduce this figure. In no cases in the current study was electron microscopy found to produce misleading findings in respect of the final diagnosis.

With respect to clinical findings there were no patterns of presentation where electron microscopy was found to be unhelpful. Therefore, it is the opinion of the authors that attempts should be made to obtain sufficient biopsy material to ensure that electron microscopy studies are carried out should this be required. However, after the light and immunofluorescence findings are known, electron microscopy was found to make no further contribution to the diagnosis of end stage or tubulointerstitial renal disease $(8 \%$ of cases in this series) (table 1), and in this instance electron microscopy may be considered unnecessary.

Electron microscopy made little contribution in the further evaluation of ischaemic hypertensive cases (table 1), but it did have a confirmatory role in some instances and in one case was considered essential.

1 Muehrcke RC, Mandal AK, Goioff SP, Isaacs EW, Volini FI. The clinical value of electron microscopy in renal disease. Arch Intern Med 1969;124:170-1.

2 Tighe JR, Jones NF. The diagnostic value of routine electron microscopy of renal biopsies. Proc Roy Soc Med 1970;63:475-7.

3 Ben-Bassat M, Stark H, Robson M, Rosenfield J. Value of routine electron microscopy in the differential diagnosis of the nephrotic syndrome. Pathol Microbiol 1974;41: $26-40$.

4 Dische FE, Parsons V. Experience in the diagnosis of glomerulonephritis using combined light microscopical, ultrastructural and immunofluorescence techniques-an analysis of 134 cases. Histopathology 1977;1:331-62.

5 Spargo BH. Practical use of electron microscopy for the diagnosis of glomerular disease. Hum Pathol 1975;6: 405-20.

6 Siegel NJ, Spargo BH, Kashgarian M, Hayslett JP. An evaluation of routine electron microscopy in the examination of renal biopsies. Nephron 1973;10:209-15.

7 Collan Y, Klockars M, Heino M. Revision of light-microscopic kidney biopsy diagnosis in glomerular disease. Nephron 1978;20:24-31.

8 Skjorten F, Halvorsen S. A study of the value of resinembedded semi-thin sections and electron microscopy in the diagnosis of renal biopsies. Acta Pathol Microbiol Scand 1981;89:257-62. 